\title{
Supramolecular Interactions of Group VI Metal Carbonyl Complexes: the Facilitating Role of 1,3-bis(p-isocyanophenyl)urea
}

Shaun Millard, ${ }^{\dagger}$ Jenny W. Fothergill, ${ }^{\star}$ Zoe Anderson, ${ }^{\dagger}$ Eric. C. Brown,,$^{\dagger}$ Matthew D. King, ${ }^{\dagger *}$ and Adam C. Colson ${ }^{\dagger *}$

†Department of Chemistry and Biochemistry, Boise State University, Boise, Idaho 83725 United States

¥Micron School of Materials Science and Engineering, Boise State University, Boise, Idaho 83725 United States

* Email: adamcolson@boisestate.edu

\section{Supporting Information}




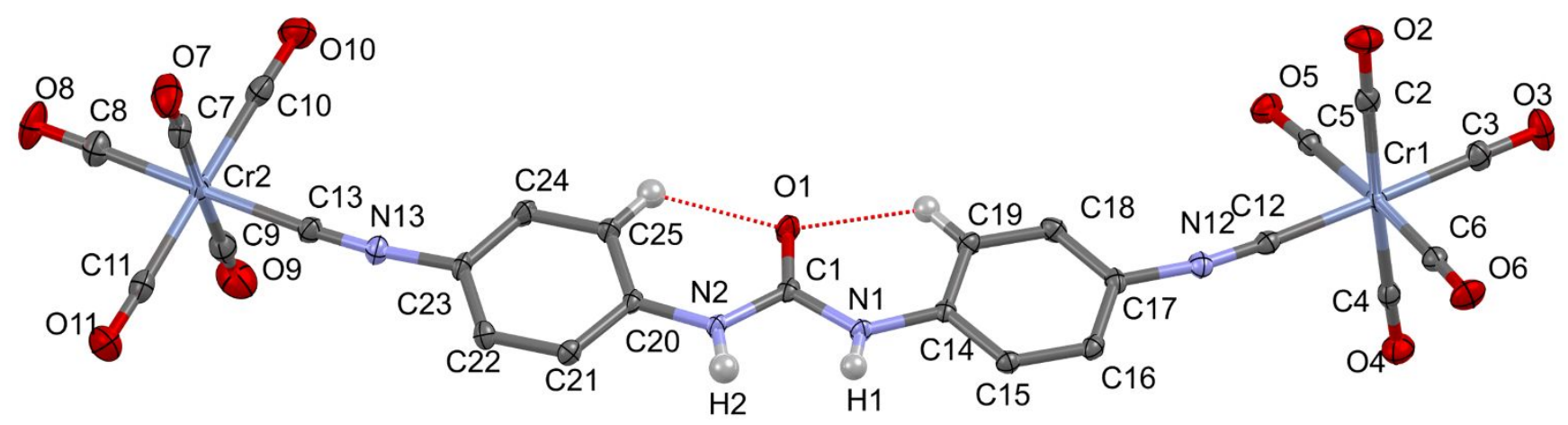

Figure S1. Molecular structure of complex 2. Thermal ellipsoids are rendered at the 50\% probability level. Only urea $\mathrm{N}-\mathrm{H}$ atoms and aromatic hydrogen atoms participating in hydrogen bonding are shown.

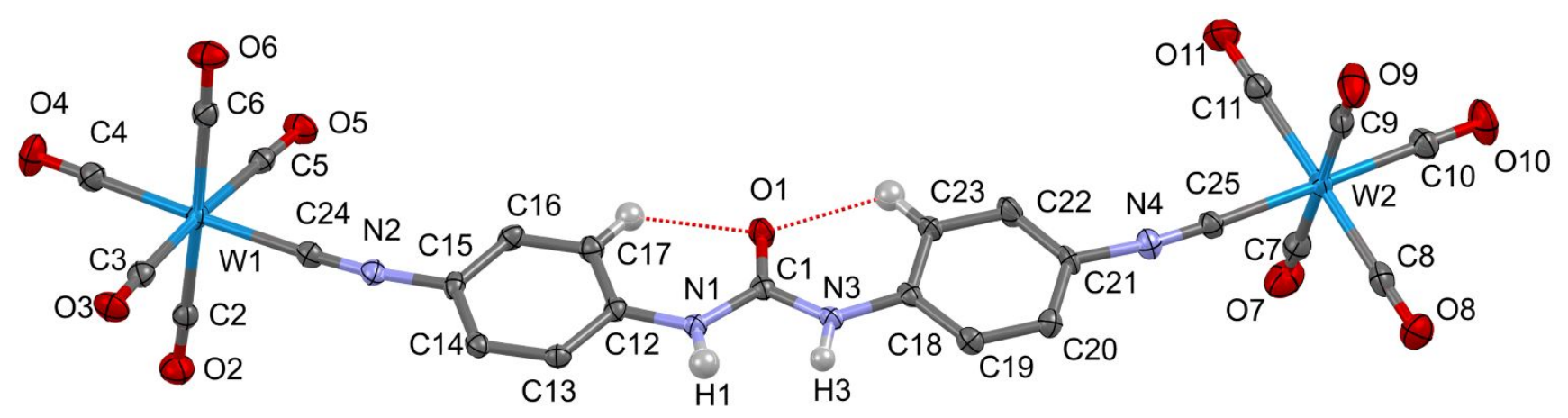

Figure S2. Molecular structure of complex 4. Thermal ellipsoids are rendered at the 50\% probability level. Only urea $\mathrm{N}-\mathrm{H}$ atoms and aromatic hydrogen atoms participating in hydrogen bonding are shown. 
Electrochemical Measurements. Cyclic voltammograms were recorded in $0.1 \mathrm{M}\left[\mathrm{Bu}_{4} \mathrm{~N}\right]\left[\mathrm{PF}_{6}\right]$ DMF solutions at $v=100 \mathrm{mV} / \mathrm{sec}$ with a Princeton Applied Research VersaSTAT 3 potentiostat. All experiments were performed using a standard three-electrode configuration under an atmosphere of pure nitrogen. Glassy carbon working electrodes ( $3 \mathrm{~mm}, \mathrm{CH}$ Instruments) were used for all measurements and were polished with aqueous slurries of $0.3 \mu \mathrm{m}$ and $0.05 \mu \mathrm{m}$ alumina powder, sequentially. After polishing, the electrodes were rinsed with Milli-Q water, methanol, and dichloromethane and dried in a stream of air. Working electrodes were preconditioned by performing three cyclical scans from 2.0 to $-2.5 \mathrm{~V}$ at $250 \mathrm{mV} / \mathrm{sec}$ in a DMF solution of $\left[\mathrm{Bu}_{4} \mathrm{~N}\right]\left[\mathrm{PF}_{6}\right]$ $(0.1 \mathrm{M})$. A graphite rod served as the counter electrode and a silver wire immersed in a $0.1 \mathrm{M} \mathrm{DMF}$ solution of $\left[\mathrm{Bu}_{4} \mathrm{~N}\right]\left[\mathrm{PF}_{6}\right]$ and separated from the cell compartment by a porous glass frit (CoralPor 1000) was employed as a $\mathrm{Ag}^{+} / \mathrm{Ag}$ pseudoreference electrode. Measured potentials are reported relative to the ferrocenium $(1+) /$ ferrocene $(0)$ redox couple, which was achieved by addition of ferrocene at the end of each set of scans.

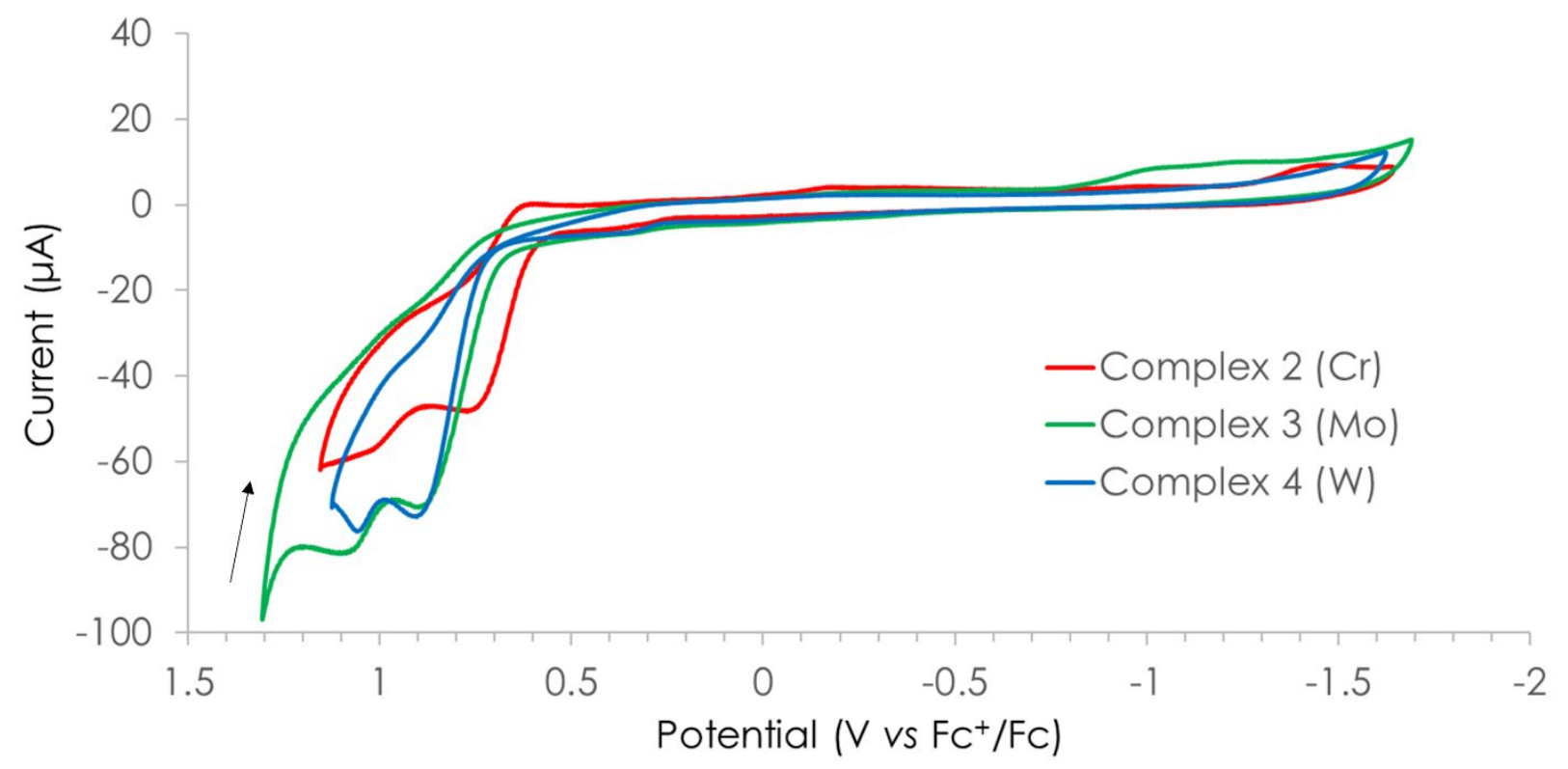

Figure S3. Cyclic voltammograms of complexes 2-4 $(\approx 1 \mathrm{mM})$ recorded in $0.1 \mathrm{M}\left[\mathrm{Bu}_{4} \mathrm{~N}\right]\left[\mathrm{PF}_{6}\right]$ DMF solution at $v=100 \mathrm{mV} / \mathrm{sec}$ with a glassy carbon working electrode, graphite rod counter electrode, and a silver wire pseudoreference electrode. 
Table S1. X-ray data collection and refinement parameters for complexes 2-4.

\begin{tabular}{|c|c|c|c|}
\hline Compound & 2 & 3 & 4 \\
\hline Formula & $\begin{array}{c}\mathrm{C}_{25} \mathrm{H}_{10} \mathrm{~N}_{4} \mathrm{O}_{11} \mathrm{Cr}_{2} \\
\cdot \mathrm{CH}_{3} \mathrm{CN}\end{array}$ & $\begin{array}{c}\mathrm{C}_{25} \mathrm{H}_{10} \mathrm{~N}_{4} \mathrm{O}_{11} \mathrm{Mo}_{2} \\
\cdot \mathrm{CH}_{3} \mathrm{CN}\end{array}$ & $\begin{array}{c}\mathrm{C}_{25} \mathrm{H}_{10} \mathrm{~N}_{4} \mathrm{O}_{11} \mathrm{~W}_{2} \\
\cdot \mathrm{CH}_{3} \mathrm{CN}\end{array}$ \\
\hline Formula weight & 687.42 & 775.30 & 951.12 \\
\hline Temperature (K) & 100 & 100 & 100 \\
\hline Crystal system & monoclinic & monoclinic & monoclinic \\
\hline Space group & $\mathrm{P} 2_{1} / \mathrm{c}$ & $\mathrm{P} 2_{1} / \mathrm{c}$ & $\mathrm{P} 2{ }_{1} / \mathrm{c}$ \\
\hline$a(\AA)$ & $6.8126(2)$ & $6.8694(5)$ & $6.8575(4)$ \\
\hline$b(\AA)$ & $13.8536(5)$ & $14.0047(10)$ & 13.9691(9) \\
\hline$c(\AA)$ & $32.1439(11)$ & $32.536(2)$ & $32.525(2)$ \\
\hline$\alpha(\operatorname{deg})$ & 90 & 90 & 90 \\
\hline$\beta(\operatorname{deg})$ & $93.8630(10)$ & $93.115(2)$ & $93.145(2)$ \\
\hline$\gamma(\operatorname{deg})$ & 90 & 90 & 90 \\
\hline Volume $\left(\AA^{3}\right)$ & $3026.82(17)$ & $3125.5(4)$ & $3110.9(3)$ \\
\hline $\mathrm{Z}$ & 4 & 4 & 4 \\
\hline density $\left(\mathrm{g} / \mathrm{cm}^{3}\right)$ & 1.509 & 1.648 & 2.031 \\
\hline abs coeff $\left(\mathrm{mm}^{-1}\right)$ & 0.784 & 0.867 & 7.454 \\
\hline $\mathrm{F}(000)$ & 1384 & 1528 & 1784 \\
\hline Crystal size (mm) & $0.42 \times 0.18 \times 0.12$ & $0.4 \times 0.05 \times 0.05$ & $0.17 \times 0.14 \times 0.05$ \\
\hline$\lambda(\mathrm{MoK} \alpha)(\AA)$ & 0.71073 & 0.71073 & 0.71073 \\
\hline $2 \theta$ range $(\mathrm{deg})$ & 5.87 to 55.068 & 5.818 to 61.12 & 5.804 to 54.968 \\
\hline reflns (coll) & 40623 & 120953 & 50308 \\
\hline reflns (unique) & 6937 & 9565 & 7120 \\
\hline Data/restraints/parameters & $6937 / 0 / 415$ & $9565 / 0 / 415$ & $7120 / 0 / 415$ \\
\hline GOF $\left(\right.$ on $\left.\mathrm{F}^{2}\right)$ & 1.131 & 1.072 & 1.206 \\
\hline Final $R$ indexes $[I \geq 2 \sigma(I)]$ & $\begin{array}{c}\mathrm{R}_{1}=0.0356 \\
\mathrm{wR}_{2}=0.0848\end{array}$ & $\begin{array}{c}\mathrm{R}_{1}=0.0327 \\
\mathrm{wR}_{2}=0.0578\end{array}$ & $\begin{array}{c}\mathrm{R}_{1}=0.0242, \\
\mathrm{wR}_{2}=0.0463\end{array}$ \\
\hline Final $\mathrm{R}$ indexes [all data] & $\begin{array}{c}\mathrm{R}_{1}=0.0433 \\
\mathrm{wR}_{2}=0.0877\end{array}$ & $\begin{array}{c}\mathrm{R}_{1}=0.0555 \\
\mathrm{wR}_{2}=0.0623\end{array}$ & $\begin{array}{c}\mathrm{R}_{1}=0.0312 \\
\mathrm{wR}_{2}=0.0477\end{array}$ \\
\hline Largest diff. peak/hole (e $\left.\AA^{-3}\right)$ & $0.45 /-0.23$ & $0.58 /-0.47$ & $0.88 /-0.43$ \\
\hline
\end{tabular}




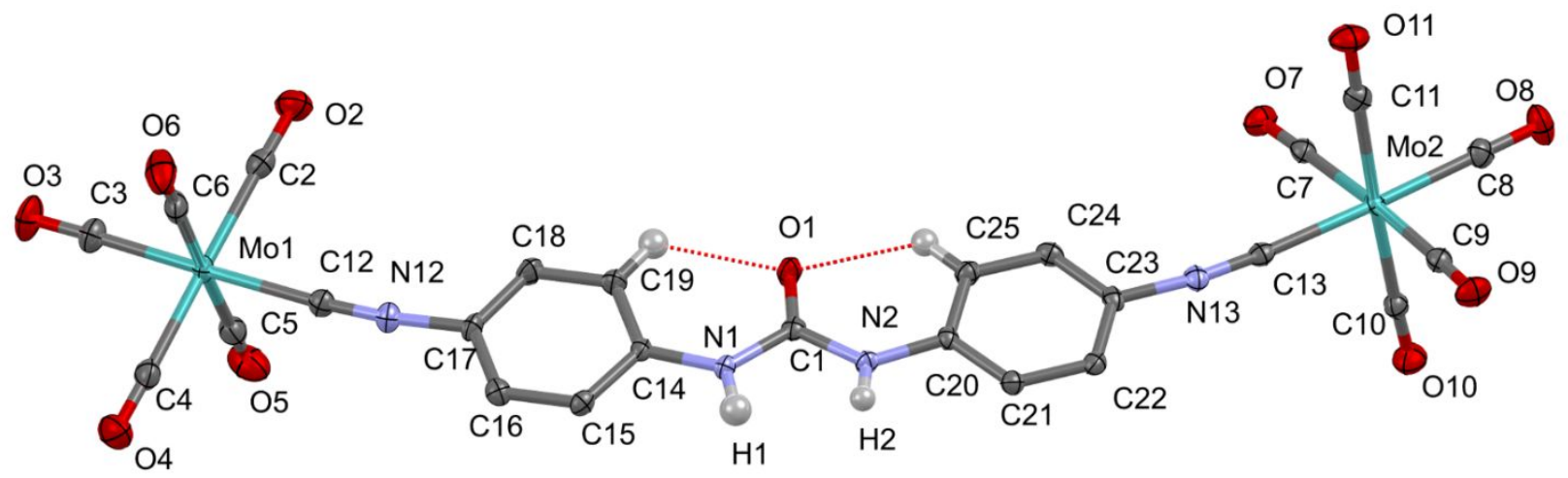

Figure S4. Atom labels used in structure analysis of complex $\mathbf{3}$ provided in Tables $\mathbf{S 2}$, S3, and S4.

Table S2. Heavy atom bond lengths $(\AA)$ and RMSD for experimental X-ray and calculated crystal structures of complex $\mathbf{3}$.

\begin{tabular}{lcc} 
Bond & exp. & DFT \\
\hline C12-Mo1 & 2.127 & 2.1778 \\
C14-C15 & 1.395 & 1.4104 \\
C14-C19 & 1.398 & 1.4094 \\
C15-C16 & 1.381 & 1.3892 \\
C16-C17 & 1.386 & 1.4013 \\
C17-C18 & 1.385 & 1.4021 \\
C18-C19 & 1.384 & 1.3907 \\
C20-C21 & 1.401 & 1.4084 \\
C20-C25 & 1.394 & 1.4085 \\
C21-C22 & 1.378 & 1.3885 \\
C22-C23 & 1.383 & 1.4003 \\
C23-C24 & 1.390 & 1.4038 \\
C24-C25 & 1.378 & 1.3904 \\
C26-C27 & 1.453 & 1.4564 \\
Mo1-C2 & 2.052 & 2.1024 \\
Mo1-C3 & 2.020 & 2.0675 \\
Mo1-C4 & 2.056 & 2.1078 \\
Mo1-C5 & 2.063 & 2.1201 \\
Mo1-C6 & 2.046 & 2.0903 \\
Mo2-C10 & 2.053 & 2.0989 \\
Mo2-C11 & 2.052 & 2.0963 \\
Mo2-C13 & 2.130 & 2.1826 \\
Mo2-C7 & 2.044 & 2.0925 \\
Mo2-C8 & 2.022 & 2.0745 \\
\end{tabular}




\begin{tabular}{lll} 
Mo2-C9 & 2.068 & 2.1158 \\
$\mathrm{~N} 1-\mathrm{C} 1$ & 1.381 & 1.3827 \\
$\mathrm{~N} 1-\mathrm{C} 14$ & 1.397 & 1.3956 \\
$\mathrm{~N} 12-\mathrm{C} 12$ & 1.155 & 1.1747 \\
$\mathrm{~N} 12-\mathrm{C} 17$ & 1.400 & 1.3832 \\
$\mathrm{~N} 13-\mathrm{C} 13$ & 1.161 & 1.1749 \\
$\mathrm{~N} 13-\mathrm{C} 23$ & 1.396 & 1.3825 \\
$\mathrm{~N} 2-\mathrm{C} 1$ & 1.373 & 1.3840 \\
$\mathrm{~N} 2-\mathrm{C} 20$ & 1.397 & 1.3958 \\
$\mathrm{~N} 3-\mathrm{C} 26$ & 1.136 & 1.1619 \\
$\mathrm{O} 1-\mathrm{C} 1$ & 1.216 & 1.2293 \\
$\mathrm{O} 10-\mathrm{C} 10$ & 1.135 & 1.1509 \\
$\mathrm{O} 11-\mathrm{C} 11$ & 1.137 & 1.1506 \\
O2-C2 & 1.137 & 1.1495 \\
$\mathrm{O} 3-\mathrm{C} 3$ & 1.143 & 1.1560 \\
$\mathrm{O} 4-\mathrm{C} 4$ & 1.138 & 1.1488 \\
O5-C5 & 1.138 & 1.1472 \\
O6-C6 & 1.138 & 1.1524 \\
O7-C7 & 1.137 & 1.1496 \\
O8-C8 & 1.147 & 1.1550 \\
O9-C9 & 1.134 & 1.1463 \\
\hline RMSD & & $\mathbf{0 . 0 2 7 9}$
\end{tabular}

Table S3. Heavy atom bond angles $\left(^{\circ}\right)$ and RMSD for experimental X-ray and calculated crystal structures of complex 3 .

\begin{tabular}{lrr} 
Bond Angle & exp. & DFT \\
\hline C1-N1-C14 & 127.10 & 127.47 \\
C1-N2-C20 & 128.00 & 128.30 \\
C10-Mo2-C11 & 176.44 & 178.47 \\
C10-Mo2-C13 & 92.66 & 91.79 \\
C11-Mo2-C13 & 90.38 & 89.43 \\
C12-N12-C17 & 172.00 & 170.06 \\
C13-N13-C23 & 171.90 & 171.30 \\
C14-C15-C16 & 120.90 & 120.95 \\
C14-C19-C18 & 119.70 & 119.87 \\
C15-C14-C19 & 119.30 & 119.19 \\
C15-C16-C17 & 119.10 & 119.29 \\
C16-C17-C18 & 120.80 & 120.38 \\
C17-C18-C19 & 120.10 & 120.30 \\
C2-Mo1-C12 & 86.43 & 88.10 \\
C2-Mo1-C3 & 92.27 & 91.92
\end{tabular}




\begin{tabular}{|c|c|c|}
\hline C2-Mo1-C4 & 178.49 & 177.19 \\
\hline C2-Mo1-C5 & 89.50 & 89.71 \\
\hline C2-Mo1-C6 & 89.54 & 87.64 \\
\hline $\mathrm{C} 20-\mathrm{C} 21-\mathrm{C} 22$ & 120.90 & 121.04 \\
\hline $\mathrm{C} 20-\mathrm{C} 25-\mathrm{C} 24$ & 119.50 & 119.55 \\
\hline $\mathrm{C} 21-\mathrm{C} 20-\mathrm{C} 25$ & 119.50 & 119.37 \\
\hline $\mathrm{C} 21-\mathrm{C} 22-\mathrm{C} 23$ & 119.00 & 119.24 \\
\hline $\mathrm{C} 22-\mathrm{C} 23-\mathrm{C} 24$ & 120.80 & 120.23 \\
\hline $\mathrm{C} 23-\mathrm{C} 24-\mathrm{C} 25$ & 120.40 & 120.56 \\
\hline C3-Mo1-C12 & 178.49 & 177.46 \\
\hline C3-Mo1-C4 & 88.90 & 90.00 \\
\hline C3-Mo1-C5 & 89.89 & 89.28 \\
\hline C3-Mo1-C6 & 90.82 & 91.79 \\
\hline C4-Mo1-C12 & 92.41 & 90.08 \\
\hline C4-Mo1-C5 & 91.45 & 92.36 \\
\hline C4-Mo1-C6 & 89.49 & 90.26 \\
\hline C5-Mo1-C12 & 89.33 & 88.18 \\
\hline C5-Mo1-C6 & 178.83 & 177.17 \\
\hline C6-Mo1-C12 & 89.94 & 90.74 \\
\hline C7-Mo2-C10 & 89.96 & 90.02 \\
\hline C7-Mo2-C11 & 88.42 & 89.14 \\
\hline C7-Mo2-C13 & 85.38 & 85.77 \\
\hline C7-Mo2-C8 & 92.48 & 91.77 \\
\hline C7-Mo2-C9 & 175.60 & 175.80 \\
\hline C8-Mo2-C10 & 87.78 & 88.11 \\
\hline C8-Mo2-C11 & 89.12 & 90.64 \\
\hline C8-Mo2-C13 & 177.82 & 177.54 \\
\hline C8-Mo2-C9 & 91.83 & 92.40 \\
\hline C9-Mo2-C10 & 91.10 & 90.63 \\
\hline C9-Mo2-C11 & 90.76 & 90.29 \\
\hline C9-Mo2-C13 & 90.30 & 90.06 \\
\hline Mo1-C12-N12 & 175.60 & 176.06 \\
\hline $\mathrm{Mo1}-\mathrm{C} 2-\mathrm{O} 2$ & 178.40 & 177.36 \\
\hline $\mathrm{Mo1}-\mathrm{C} 3-\mathrm{O} 3$ & 178.50 & 177.88 \\
\hline Mo1-C4-O4 & 178.60 & 178.49 \\
\hline Mo1-C5-O5 & 178.70 & 177.70 \\
\hline Mo1-C6-O6 & 179.20 & 177.40 \\
\hline $\mathrm{Mo} 2-\mathrm{C} 10-\mathrm{O} 10$ & 176.70 & 177.09 \\
\hline Mo2-C11-O1 & 178.00 & 179.22 \\
\hline Mo2-C13-N13 & 175.50 & 174.57 \\
\hline Mo2-C7-O7 & 176.60 & 176.05 \\
\hline $\mathrm{Mo} 2-\mathrm{C} 8-\mathrm{O} 8$ & 179.20 & 178.48 \\
\hline
\end{tabular}




\begin{tabular}{llr} 
Mo2-C9-O9 & 178.70 & 177.70 \\
N1-C1-N2 & 111.50 & 111.70 \\
N1-C14-C15 & 117.00 & 116.85 \\
N1-C14-C19 & 123.70 & 123.96 \\
N12-C17-C16 & 120.30 & 120.85 \\
N12-C17-C18 & 118.90 & 118.76 \\
N13-C23-C22 & 120.50 & 120.81 \\
N13-C23-C24 & 118.70 & 118.96 \\
N2-C20-C21 & 116.70 & 116.54 \\
N2-C20-C25 & 123.80 & 124.09 \\
N3-C26-C27 & 178.80 & 179.85 \\
O1-C1-N1 & 124.10 & 124.39 \\
O1-C1-N2 & 124.40 & 123.91 \\
\hline RMSD & & $\mathbf{0 . 8 6}$
\end{tabular}

Table S4. Heavy atom dihedral angles $\left(^{\circ}\right)$ and RMSD for experimental X-ray and calculated crystal structures of complex $\mathbf{3}$.

\begin{tabular}{lrr} 
Dihedral Angle & \multicolumn{1}{c}{ exp. } & \multicolumn{1}{c}{ DFT } \\
\hline $\mathrm{C} 1-\mathrm{N} 1-\mathrm{C} 14-\mathrm{C} 15$ & 173.1 & -179.16 \\
$\mathrm{C} 1-\mathrm{N} 1-\mathrm{C} 14-\mathrm{C} 19$ & -7.5 & 1.15 \\
$\mathrm{C} 1-\mathrm{N} 2-\mathrm{C} 20-\mathrm{C} 21$ & 179.3 & 177.82 \\
$\mathrm{C} 1-\mathrm{N} 2-\mathrm{C} 20-\mathrm{C} 25$ & -0.5 & -2.82 \\
$\mathrm{C} 14-\mathrm{C} 15-\mathrm{C} 16-\mathrm{C} 17$ & 0.1 & 0.19 \\
$\mathrm{C} 14-\mathrm{N} 1-\mathrm{C} 1-\mathrm{N} 2$ & -179.2 & 177.72 \\
$\mathrm{C} 14-\mathrm{N} 1-\mathrm{C} 1-\mathrm{O} 1$ & 1.0 & -2.02 \\
$\mathrm{C} 15-\mathrm{C} 14-\mathrm{C} 19-\mathrm{C} 18$ & -3.2 & -1.12 \\
$\mathrm{C} 15-\mathrm{C} 16-\mathrm{C} 17-\mathrm{C} 18$ & -2.8 & -0.83 \\
$\mathrm{C} 15-\mathrm{C} 16-\mathrm{C} 17-\mathrm{N} 12$ & 176.2 & 178.44 \\
$\mathrm{C} 16-\mathrm{C} 17-\mathrm{C} 18-\mathrm{C} 19$ & 2.5 & 0.50 \\
$\mathrm{C} 17-\mathrm{C} 18-\mathrm{C} 19-\mathrm{C} 14$ & 0.5 & 0.49 \\
$\mathrm{C} 19-\mathrm{C} 14-\mathrm{C} 15-\mathrm{C} 16$ & 2.9 & 0.79 \\
$\mathrm{C} 20-\mathrm{C} 21-\mathrm{C} 22-\mathrm{C} 23$ & -0.2 & -0.06 \\
$\mathrm{C} 20-\mathrm{N} 2-\mathrm{C} 1-\mathrm{N} 1$ & -178.0 & 179.74 \\
$\mathrm{C} 20-\mathrm{N} 2-\mathrm{C} 1-\mathrm{O} 1$ & 1.9 & -0.52 \\
$\mathrm{C} 21-\mathrm{C} 20-\mathrm{C} 25-\mathrm{C} 24$ & 0.5 & 1.20 \\
$\mathrm{C} 21-\mathrm{C} 22-\mathrm{C} 23-\mathrm{C} 24$ & 0.0 & 0.55 \\
$\mathrm{C} 21-\mathrm{C} 22-\mathrm{C} 23-\mathrm{N} 13$ & -179.1 & -178.62 \\
$\mathrm{C} 22-\mathrm{C} 23-\mathrm{C} 24-\mathrm{C} 25$ & 0.4 & -0.16 \\
$\mathrm{C} 23-\mathrm{C} 24-\mathrm{C} 25-\mathrm{C} 20$ & -0.6 & -0.73 \\
$\mathrm{C} 25-\mathrm{C} 20-\mathrm{C} 21-\mathrm{C} 22$ & -0.1 & -0.82 \\
$\mathrm{~N} 1-\mathrm{C} 14-\mathrm{C} 15-\mathrm{C} 16$ & -177.6 & -178.92
\end{tabular}




\begin{tabular}{lrr} 
N1-C14-C19-C18 & 177.4 & 178.57 \\
N12-C17-C18-C19 & -176.5 & -178.79 \\
N13-C23-C24-C25 & 179.5 & 179.03 \\
N2-C20-C21-C22 & -179.9 & 178.57 \\
N2-C20-C25-C24 & -179.7 & -178.14 \\
\hline RMSD & & $\mathbf{2 . 4 6}$
\end{tabular}

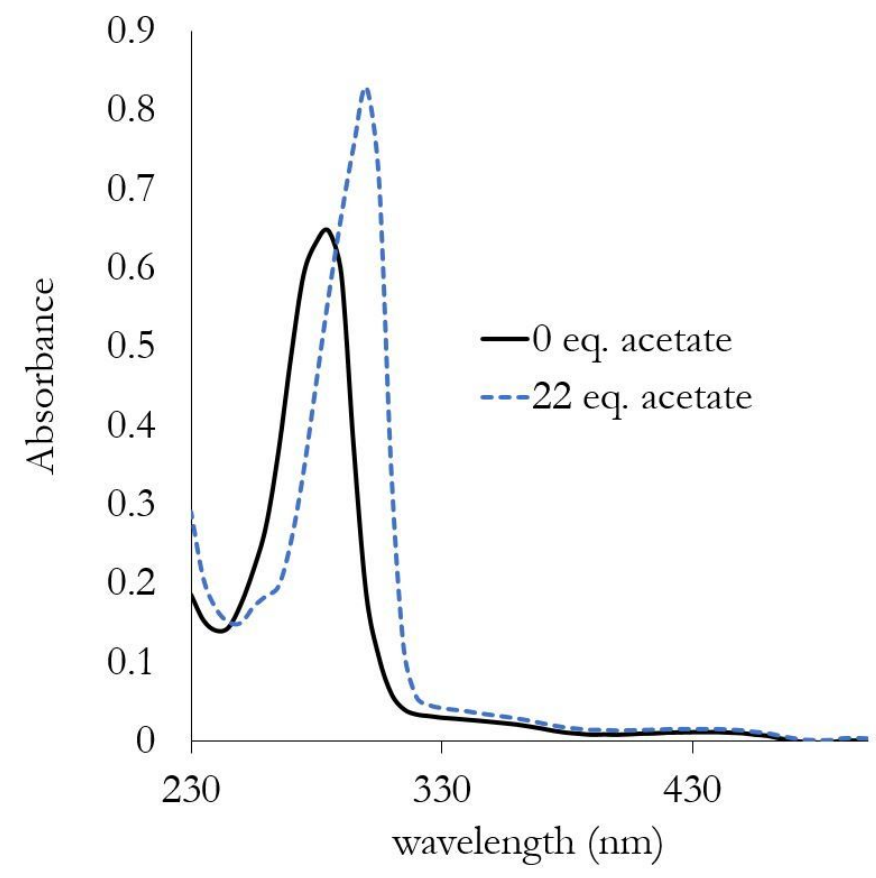

Figure S5. UV-Vis spectrum of $1\left(10.8 \mu \mathrm{M}\right.$ in $\left.\mathrm{CD}_{3} \mathrm{CN}\right)$ in the absence and presence of excess acetate anion. 

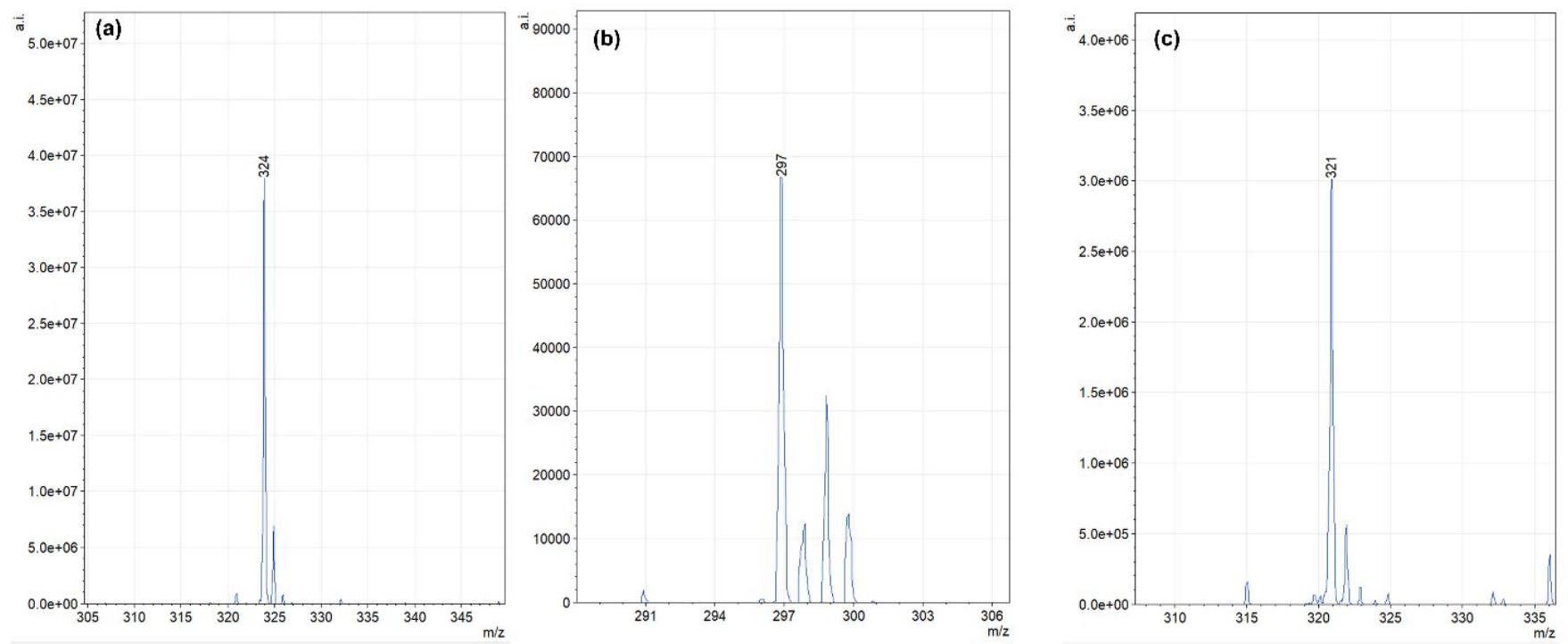

Figure S6. ESI-MS data for 1:1 host-guest complexes of 1 with (a) $\mathrm{NO}_{3}^{-}$, (b) $\mathrm{Cl}^{-}$, and (c) $\mathrm{CH}_{3} \mathrm{COO}^{-}$
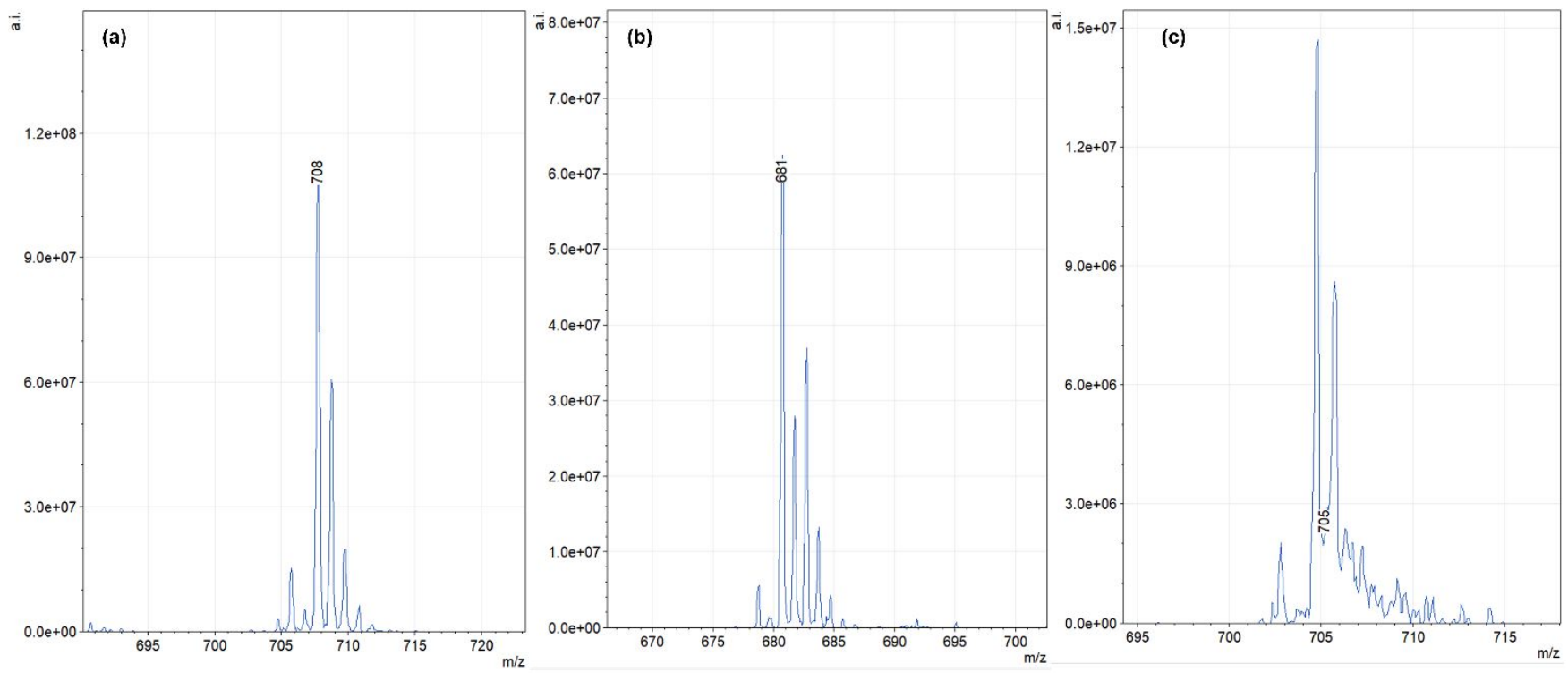

Figure S7. ESI-MS data for 1:1 host-guest complexes of 2 with (a) $\mathrm{NO}_{3}^{-}$, (b) $\mathrm{Cl}^{-}$, and (c) $\mathrm{CH}_{3} \mathrm{COO}^{-}$ 

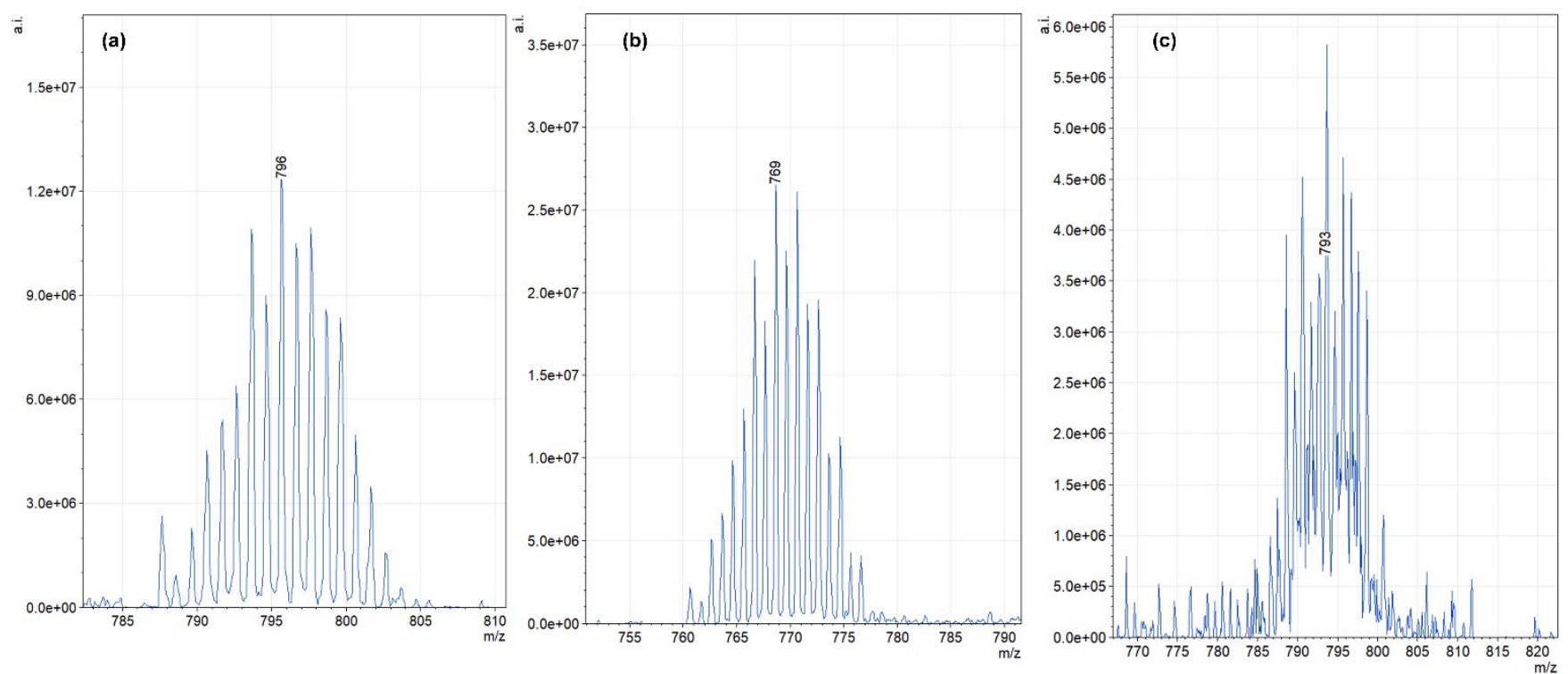

Figure S8. ESI-MS data for 1:1 host-guest complexes of 3 with (a) $\mathrm{NO}_{3}^{-}$, (b) $\mathrm{Cl}^{-}$, and (c) $\mathrm{CH}_{3} \mathrm{COO}^{-}$
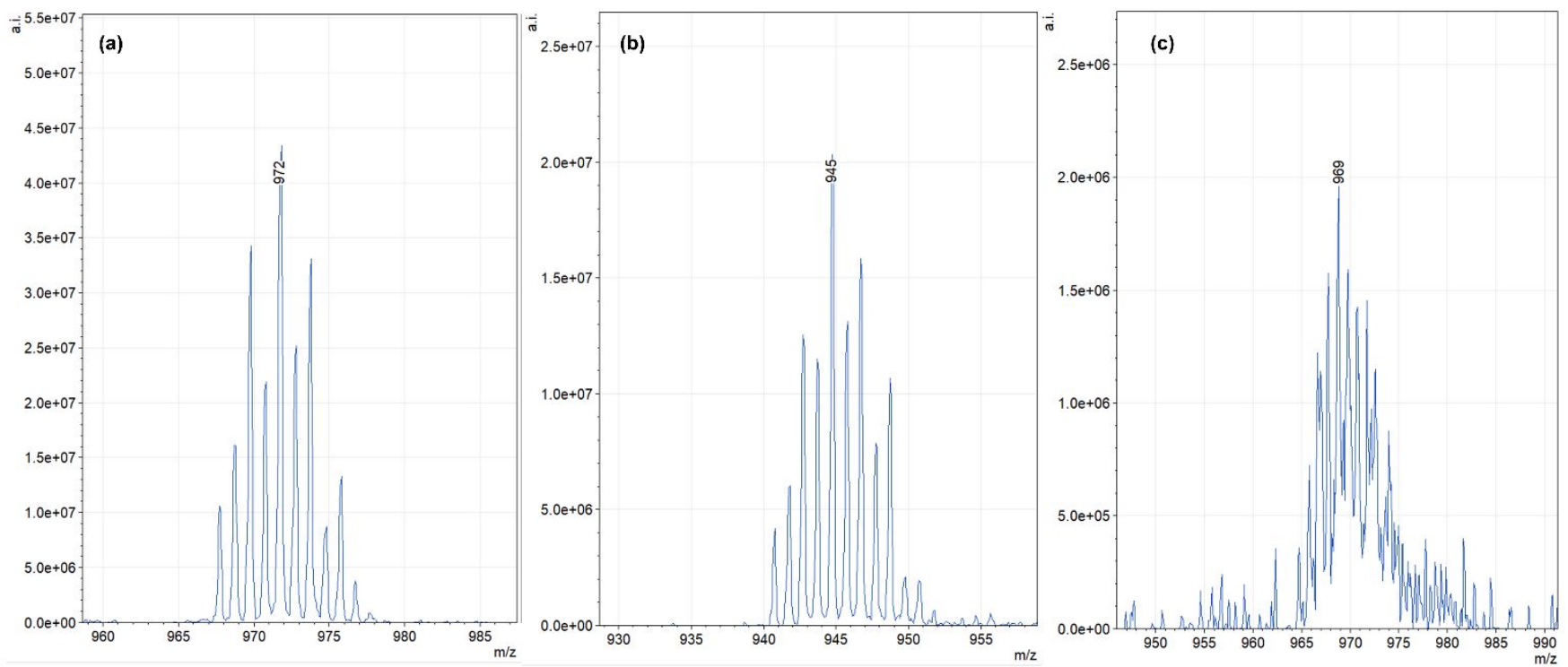

Figure S9. ESI-MS data for 1:1 host-guest complexes of 4 with (a) $\mathrm{NO}_{3}{ }^{-}$, (b) $\mathrm{Cl}^{-}$, and (c) $\mathrm{CH}_{3} \mathrm{COO}^{-}$ 
(a)

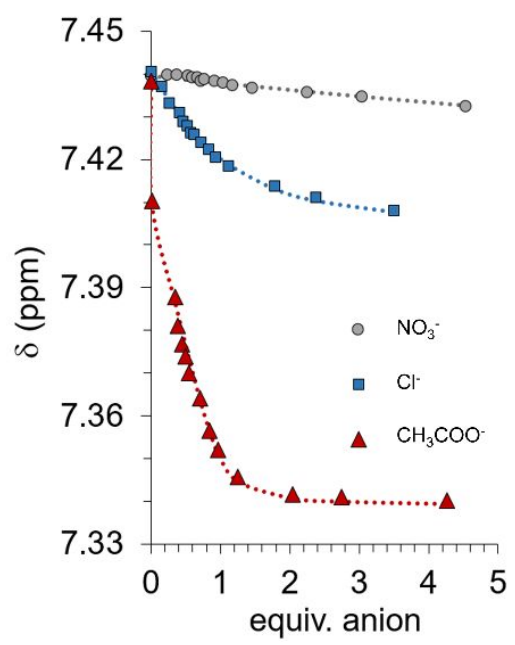

(b)

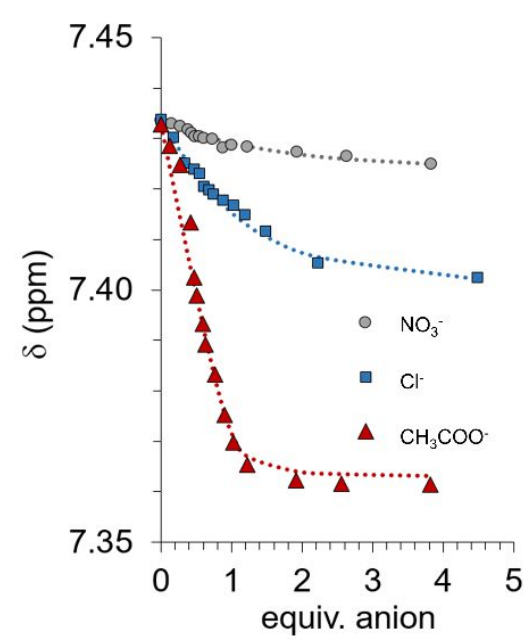

(c)

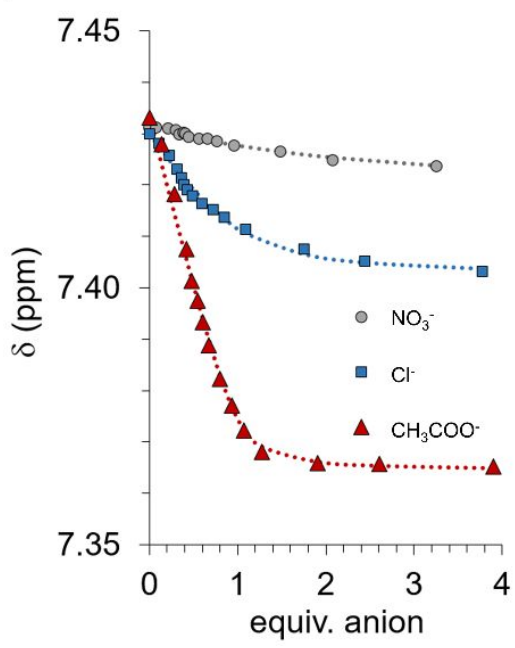

Figure S10. ${ }^{1} \mathrm{H}$ NMR chemical shifting observed during titration of (a) 2, (b) 3, and (c) 4 ( 0.1 $\mathrm{mM}$ in $\mathrm{CD}_{3} \mathrm{CN}$ ) with nitrate, chloride, and acetate anions. The dotted lines represent the results of non-linear fitting to a 1:1 host-guest binding model. 